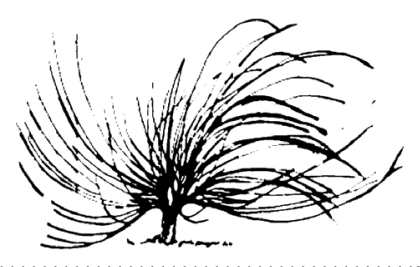

\title{
Reflexiones sobre el posconflicto: Aprendizaje de la historia
}

\author{
Fernando Arturo Romero Ospina ${ }^{1}$ \\ Institución educativa Gonzalo Jiménez de Quesada \\ Sede rural Cuaya \\ Suesca, Colombia \\ humanodelirio@yahoo.com
}

\begin{abstract}
Resumen
El siguiente ensayo reflexiona sobre cómo se debe efectuar el aprendizaje de la historia-política colombiana en la escuela, en el escenario del posconflicto, para fomentar proceso de reconciliación. Para esto, veremos cómo la historia ha tenido diversas definiciones, la cuales han estado presentes en la enseñanza de la escuela, cumpliendo un rol social y político en la construcción de una identidad y memoria oficial. Sin embargo, en el escenario del posconflicto, la escuela -como centro socialmente reconocido- debe agenciar procesos de revisión de la historia de la sociedad colombiana, para poder entender los sucesos y orígenes del conflicto actual, en aras de una reconciliación duradera donde los actores del conflicto expresen sus puntos de vista para que los efectos de la guerra no causen de nuevo un nuevo conflicto.
\end{abstract}

\section{(c) (i) (3)}

Recibido: 21 de enero de 2016-Aprobado: 25 de abril de 2016

1 Fernando Romero es licenciado en Pedagogía infantil de la Universidad Francisco José de Caldas de Bogotá, Colombia. Actualmente es maestro de la Escuela Rural Cuaya , ubicada en el municipio de Suesca. Ha escrito artículos referentes a la escuela laica en la revista Convocación de Uruguay y sobre la construcción social de la infancia en la revista Viraje de la Universidad de Caldas de Colombia. 
Palabras clave: Historia, escuela, reconciliación, postconflicto, ética, aprendizaje.

\begin{abstract}
This essay intends to reflect on how to make the learning of the political history of Colombia at school in the post-conflict scenario to encourage a reconciliation process. To do this, we will see how history has had various definitions which have been present in the school teaching fulfilling a social and political role in the construction of an identity and official memory. However, in the post conflict scenario, the school, as a socially recognized center, must facilitate a process of revision of the history of Colombian society to understand the events and origins of the current conflict so that a lasting reconciliation allows the actors of the conflict to express their points of view, and the effects of war do not cause a new conflict.
\end{abstract}

Keywords: history, school, reconciliation, post conflict, ethics, learning

\title{
Introducción
}

El objetivo de este ensayo es contribuir sobre la reflexión de los retos del posconflicto en Colombia, específicamente sobre cómo efectuar el aprendizaje de la historia en la escuela primaria, asignatura que involucra a una sociedad en específico como la colombiana, pero que debe ser referente al país que ha tenido conflictos armados y está en un proceso de posconflicto, ya que las secuelas de los conflictos perduran generando discriminación y estigmatización en la población.

En este sentido, la reflexión sobre el aprendizaje de la historia en la escuela primaria es un factor determinante en la construcción del presente y de la memoria colectiva de una sociedad en el posconflicto y significa responder: ¿por qué?, ¿cómo? y ¿para qué? se dio el conflicto y qué hacer para que esto no vuelva a suceder.

En Colombia, el enfrentamiento armado lleva más de sesenta años, si se cuenta con el suceso de la muerte de Jorge Eliecer Gaitán, acontecimiento que encendió el conflicto partidista el 9 de abril de 1948, suceso que tuvo un punto de altas y bajas, hasta el nacimiento de las guerrillas con los grupos de las FARC y ELN. 
Estos acontecimientos aún perduran en la memoria colectiva de la población. Es un conflicto extenso en el tiempo; pero de baja duración militar; los retos son grandes ya que la violencia se ha establecido como un suceso en la cotidianidad, lo cual la normaliza.

Esta cotidianidad del conflicto ha establecido unas formas de comunicarse y relacionarse entre la sociedad colombiana y ha afectado toda posibilidad de paz, ya que uno de los puntos a tratar es que la paz es una responsabilidad social.

En la actualidad, existe un proceso de paz en la Habana, con el grupo de las FARC, pero en la población hay desconfianza, ya que los procesos en Colombia han sido fallidos: el último caso fue el de San Vicente del Caguán, efectuado entre 1998 y 2002 con la consecuencia, más bien, del recrudecimiento del conflicto.

Por eso, se debe dejar claro que los procesos de paz no se cierran con una firma, sino que abren nuevas aristas a la sociedad. Una de estas aristas es la manera como se va a aprender la historia del conflicto, ya que permitirá reflexionar sobre las condiciones sociales, políticas y económicas que dieron origen al problema, haciendo de este escenario un paso para una reconciliación que perdure en las nuevas generaciones $\mathrm{y}$ en las actuales.

Para este ensayo se usa el método cualitativo de revisión bibliográfica, por esto se iniciará indicando la dificultad de definir el concepto de la historia, después cómo se ha dado la enseñanza de la historia-política en la escuela básica en Colombia, la cual tiene dos objetivos: la construcción de una memoria "oficial" y la formación de una identidad nacional en la ciudadanía y, finalmente, se indicará que el posconflicto en la escuela es una posibilidad para construir un discurso de la reconciliación en la sociedad.

\section{1. ¿Qué es la historia?}

Para iniciar la reflexión es importante establecer claridades sobre a qué se le llama historia; sobre ¿qué es la historia?, fuera de ser descontextualizada, ha sido campo de debate en la actualidad, puesto que su respuesta "refleja nuestra posición en el tiempo, y forma parte de nuestra respuesta a la pregunta, más amplia, de qué idea hemos de formarnos de la sociedad en que vivimos"(Carr, 1984, p. 11). 
Varios estudios han tratado de buscar una respuesta no definitiva sino que signifique nuevos rumbos en este conocimiento, entre quienes podemos encontrar al inglés Edward H. Carr y al francés Bloch, quienes entendieron que pensar la historia como la acumulación de hechos o la búsqueda de la verdad en el pasado, es carente de un sentido histórico, ya que se recurre a una visión determinista de la historia.

Esto se debía a dos puntos centrales: el rol del historiador o historiadora y al método que utiliza la historia y su finalidad, donde se pretendía resaltar los sucesos, acontecimientos más representativos que una sociedad había tenido para fomentar la identidad y una historia válida para la población.

Con esto, cada autor o autora interroga a quienes han escrito la historia, cuestionado su quehacer, su conocimiento y sus métodos. Carr (1984) comenta en su libro ¿Qué es la historia?, que la historia decimonónica daba veracidad a los hechos, es decir, solo mostraba lo que realmente aconteció.

Esta postura positivista estaba marcada por los avances de la ciencia y su método científico que también compartían las ciencias sociales. Estos hechos estaban ubicados en los documentos, en los sucesos contados por unos pocos, pero que eran relatos sobre reyes, personajes que tenía una importancia por su rol social e histórico.

Sin embargo, en ese discurso se pregunta: ¿qué sucesos son merecidos de ser contados? Y esta pregunta surge también sobre el papel del historiador o historiadora, como lo indica Carr al decir: "no se puede hacer historia, si el historiador no llega a establecer algún contacto con la mente de aquellos sobre los que escribe" (1984, p. 33). Esto quiere decir que el historiador o historiadora es quien construye la historia.

En conclusión, para Carr la historia era vista por los sujetos historiadores como su objeto de estudio que se pretendía ver de forma objetiva, describiendo los hechos de forma muy detallada; sin embargo, esta historia era escrita con la visión desde el presente, por esto define la historia como "un proceso continuo de interacción entre el historiador y sus hechos, un diálogo sinfín entre el presente y el pasado" (1984, p. 40).

En el caso de Bloch, para responder la pregunta sobre ¿qué es la historia?, inicia diciendo que definir la historia como "la ciencia del pasado. Me parece una forma impropia de hablar" (1952, p. 32). Esto, porque se pregunta ¿cómo puede ser objeto de un conocimiento racional, 
sin una delimitación previa, una serie de fenómenos que no tienen otro carácter común que el no ser nuestros contemporáneos? (1952).

El concepto de historia, entonces, es visto en un punto de la línea del tiempo, relacionado con la narración del pasado opuesta al presente, tratando de abstraer el tiempo para convertirlo en un objeto de estudio. Pero esta historia descansa en la preocupación de la observación, narración y descripción; pero no del ser humano.

Un ejemplo de esto es el tratar de ubicar el objeto de estudio entre historia e arqueología, la cual, según Childen (1985), es una cuestión de tiempo, donde en la historia " el periodo analizado es, cuando mucho, una centésima parte del tiempo durante el cual los hombres se han mostrado activos en nuestro planeta" (p. 19).

Por lo tanto, no se trata, entonces, de que el historiador o historiadora realice una observación de "la realidad objetiva" sino, como señala Restrepo (2000) destacando a Foucault como historiador quien "conecta el trabajo histórico y la investigación del presente". (p. 146). Con esta postura quien estudia la historia conecta su experiencia para construir un pasado.

En este sentido, Bloch (1952) plantea como "en efecto, hace mucho que nuestros grandes antepasados, un Michelet y un Fustel de Coulanges, nos habían enseñado a reconocerlo: el objeto de la historia es esencialmente el hombre" (p. 34).

Sin embargo, Bloch aclara que no es el "hombre" sino son "los hombres", el objeto de la historia son los "hombres-tiempo", esto que señala Bloch es importante, ya que considera la historia no como una entidad muerte sino que está en constante movimiento, es un discurso vivo, no del pasado muerto sino que es tiempo y que aún ejerce sus mágica presencia en el presente.

Es más, entender el pasado significa entender nuestro presente, no se trata entonces de una visión cerrada, sino que es la compresión del presente lo que permite comprender el pasado. Este cambio de postura constituye una posibilidad de ver al ser humano en constante cambio.

Siguiendo con lo anterior, esta postura sobre la historia es la respuesta de la visión decimonónica con lo cual dará inicio una nueva forma de investigación social, a la cual se le llama Escuela de los Annales, con la fundación de la revista Annales en 1929 dirigida por Henry Pirene "frente a la que se agruparon varios estudiosos que compartían 
la inquietud por un nuevo tipo de historia, no anecdótico, sino centrado en problemas" (Castro, 2008, p. 33).

Por lo tanto, viendo estas dos posturas, tanto Carr como Bloch quieren dejar la visión de una historia que se "veía como un drama representado en torno al héroe y a la acción y la personalidad de éste. Tal drama era el tema central de la obra histórica" (Jaramillo, 1999 p. 17), por una historia que se escribe desde el presente y que se debe hablar en plural y no en singular.

Es así como entendiendo el objeto de estudio de la historia, su enseñanza es fundamental en la construcción del ser humano como ser social y temporal, como lo dice Betancourt (1995): "La enseñanza de la Historia es determinante en la formación de la "conciencia cívica y democrática' de todo ciudadano y a través de ella, decisiva en la configuración social de la "conciencia histórica"' (p. 25).

Tanto la postura de Bloch como de Betancourt tienen una preocupación por la conciencia histórica, es decir, que el ciudadano o ciudadana es un sujeto temporal, reflexivo, con un origen, que necesita entender su presente para comprender el pasado. Y esto porque "el conocimiento del pasado es algo que está en constante progreso, que se transforma y se perfecciona sin cesar" (p. 59).

La historia estudia a losseres humanos; pero, como hemos indicado, reducirla a la franja del tiempo o al pasado es inapropiado, porque también cabe preguntar sobre la historia como arte y ciencia, ya que en sus narraciones también nos llevan a conocer a los hombres y mujeres, en nuestro presente.

\section{Escuela-historia: Caminos divergentes}

Ya que hemos indicado el objeto de estudio de la historia son los seres humanos-tiempo, la importancia de su aprendizaje radicara en la compresión de nuestra realidad como humanidad. Ahora ¿cómo se enseña la historia?, esta es otra pregunta que abarca la posición de una entidad tan cerrada como es la escuela.

Tradicionalmente, el aprendizaje de la historia se inicia como los cuentos en la literatura infantil "érase una vez", "había una vez", en la enseñanza de la historia-política en la escuela básica se da prioridad a los héroes y no a las comunidades "érase una vez un héroe", "había una vez dos partidos políticos", etc. 
Para esto se ha fragmentado en épocas, como dice Álvarez (1995): prehistoria, conquista, colonia, independencia y república" (p. 25) esto para recordar los momentos gloriosos de una patria, pero también como indica Álvarez (1995) "la narrativa histórica tenía por función explicar las causas del presente inmediato que se mostraba confuso" (p. 23 ).

De lo anterior se puede decir que la historia que se enseña es contada de una forma lineal, progresiva y con un discurso que busca legitimar el orden social y, en este caso, la idea de la república ya que la historia será entendida como "la gran lucha de la civilización por hacer triunfar aquellos principios originarios" (Gallego,1995, p. 24).

Es decir, la búsqueda constante de la civilización olvidando el pasado de la "barbarie", comentando en cada etapa cómo, a partir de la idea del progreso, se ha venido construyendo un proyecto de nación, comparándola con el crecimiento del ser humano, de una niñez a una adolescencia pasando etapas de crecimiento y desarrollo.

Ahora, esta enseñanza generó "una historia oficial," es decir, aquella que permite el establecimiento de un "status quo" que ha negado "otra historia" construida por los pueblos y comunidades de esta sociedad multicultural. Por lo tanto, la historia como materia de enseñanza tenía un propósito patriotero en el siglo XIX.

Por lo tanto, esta enseñanza de la historia ha permitido la existencia de un desconocimiento del pasado y presente de Colombia, peligroso fenómeno que en un artículo del periódico el Tiempo escrito por Andrea Linares, titulado: "Historia, la gran materia olvidada", hace un análisis de la enseñanza de la historia en Colombia, de la consecuencia y efectos de una formación histórica, en uno de los apartados dice:

Desconocer la historia fomenta un pensamiento mágico: hace que los estudiantes crean en mitos e ideas falsas con extrema facilidad, no tengan una visión razonable de sí mismos y sean personas manipulables. (El tiempo, 2013)

Es así como la falta de la enseñanza de la historia contextualizada alimenta la amnesia social peligrosa en una etapa de conflicto y posconflicto, porque se reduciría a un pensamiento mágico, y no al análisis del porqué hemos tomados decisiones como sociedad. Sería un escenario ideal para el olvido y la manipulación, pero ante todo para la impunidad. 
Con este panorama, el aprendizaje de la historia debe apuntar en la formación de dos habilidades, como indica Carretero y Monteranero (2008): "la capacidad de comprender el tiempo histórico y razonar causalmente, por un lado; la capacidad de valorar e interpretar críticamente las fuentes de información históricas por otro" (p. 135).

La comprensión y el desarrollo de un pensamiento crítico permitirán desarrollar una conciencia histórica, "por lo tanto, se debe pensar la Historia de manera global y vinculada al presente" (Lepe y Lima, 2014, p. 26). Encontramos estrategias como las que se discuten en México en el Instituto Nacional para la evaluación de la Educación (INEE), que crea estrategias de comprensión lectora donde establezcan relaciones entre el pasado y el presente, en lecturas globales.

Otro ejemplo es la discusión que se da en Estados Unidos sobre la historia, ya que el Comité Bradley y la Comisión Nacional de Estudios Sociales indican que "las escuelas no pueden seguir tratando el currículo de historia como la cobertura y la memorización de una gran cantidad de fechas y nombres" (Perrone, p. 57, 1999).

Para cambiar esta situación se desarrollan tópicos partiendo del conocimiento que tienen los estudiantes, de sus experiencias vinculándolas con situaciones concretas, donde el estudiante es desafiado en su indagación a partir de preguntas y temas específicos.

En este caso, la comisión habla de tres periodos que deben ser estudiados en secundaria, por ejemplo: historia y geografía mundial y estadounidense hasta 1750 , historia y geografia mundial y estadounidense 1750-1900 y, finalmente, historia y geografía mundial y estadounidense desde 1900 (Perrone,1999).

De lo anterior se desprende que la escuela no es un agente pasivo sino, como señala Álvarez (1995), "la escuela es un acontecimiento" (p. 10), es decir, la escuela no es una institución material sino cultural, donde los procesos históricos, sociales, políticos y económicos generan cambios en el aprendizaje, socialmente legitimados para la educación.

La escuela cumple una función muy interesante: educar. El tema de la educación es muy variado. Desde la pedagogía y psicología y demás áreas del conocimiento se ha abordado; sin embargo, siempre queda en el aire el ¿para qué de la educación? Una respuesta simplista sería que la educación permitiría, con el tiempo, la integración de los individuos a la sociedad para el cumpliendo de unos roles, pero aún queda la pregunta abierta. 
“Cuando se pregunta 'educación ¿para qué?' este 'para qué' no resulta ya inteligible por sí mismo, no forma parte sin mayor dificultad de lo cotidianamente asumido y operante, todo se ve afectado por la inseguridad y exige reflexiones no precisamente fáciles" (Adorno, 1998, p. 94). Por lo tanto, debemos partir del dilucidar las inseguridades y reflexionar sobre el para qué.

También la educación conlleva tiempo, estrategias y objetivos. Para que el ser humano tome ciertos elementos, si se quiere herramientas como es la lectura, escritura y toda un acervo cultural, necesita tiempo, para que su estadía en el mundo sea placentera, esto conlleva a reflexionar sobre cómo vemos a este sujeto, si en forma pasiva o activa.

En este sentido, la educación no es un elemento estático, ya que "la educación es cosa social; es decir, que pone, en contacto al niño con una sociedad determinada, y no con la sociedad in genere" (Durkheim, 1976, p. 15) y el lugar privilegiado socialmente es la escuela que se ha establecido para que las disciplinas y métodos se hagan presentes en prácticas pedagógicas y cumplan con su papel educativo.

En efecto, la escuela es una institución que se ha consolidado con el tiempo y "tal legitimación es particularmente porque es un vínculo entre la estructura económica y social y la mente de los niños, futura fuerza de trabajo y participantes políticos" (Carnoy, 2000, p. 13); por lo tanto, este sistema es perfecto para legitimar ciertos proceso y, con la enseñanza de la historia, se pueden reforzar estos elementos.

Para esto la escuela "hace propia la cultura particular de las clases dominantes, enmascara su naturaleza social y la presenta como la cultura objetiva, indiscutible, rechazando al mismo tiempo las culturas de los otros grupos sociales. La escuela, legitima de tal manera la arbitrariedad cultural" (Bourdieu, 1995, p. 18) que permite que el orden social se establezca y se siga con una estructura social.

La anterior aseveración implica que en las escuelas se realizab acciones, pero "toda acción pedagógica es objetivamente una violencia simbólica en cuanto impone, a través de un poder arbitrario, una arbitrariedad cultural" (Bourdieu, 1995, p. 25); es decir, se efectúa una violencia para imponer significado $\mathrm{y}$, en este sentido, legitima el poder pedagógico como instrumento de formación para establecer un control social y una ideología. 
Por lo tanto, como lo dice Betancourt (1995, p. 30):

No existe una educación neutra, una transmisión de hechos históricos e interpretación exenta de una determinada comprensión de la sociedad generalmente admitida como normal, como algo común y corriente, la cual de hecho implica unas determinadas concepciones económicas, sociales, políticas y culturales.

En este sentido, la ideología que genera el proceso educativo marcará un tipo de sociedad, en los términos de Van DIjk quien dice:

Las ideologías no son cualquier tipo de creencias socialmente compartidas, como el conocimiento sociocultural o las actitudes sociales, sino que son más fundamentales o axiomáticas. Ellas controlan y organizan otras creencias socialmente compartidas. (2005, p. 9-36)

Debemos entender que la ideología no negaría la acción de cada sujeto o su individualidad, pero en la relación entre posconflicto, conciencia-histórica y escuela, la ideología que se comunique en forma pedagógica, sin dar cabida a una naturalización de fenómenos y procesos sociales.

Entonces, la relación entre ideología e historia debe ser sujeta a estudio en la escuela, como lo indica Alaín Touraine (1997):

La difficulté de notre täche presente est précisément de découvrir une societé et une cultura en formation, sans recourir a une prétendue nécessité historique, á un sens de la histoire [La dificultad de nuestra tarea es precisamente la presencia de un descubrimiento de la sociedad y la formación del sector cultura, sin tener que recurrir a una supuesta necesidad histórica, a un sentido de la historia] (p. 71)

Por otra parte, esta formación debe alcanzar la conciencia histórica como la posibilidad del manejo de la información donde:

Lo primero que los alumnos deben reconocer es que un hecho o proceso en la Historia no es unicausal, sino que se produce por múltiples y diversas causas que tiene lugar en un tiempo y espacio determinado. (Lepe y Lima, 2014, p. 16) 
Las causas de los sucesos y sus múltiples consecuencias es la posibilidad de una lectura global a partir de relatos históricos, memorial oral, pictogramas, reconstrucción de la memoria colectiva; ello permitirá que el conflicto histórico se vea críticamente, es decir, de forma en donde las partes puedan ser escuchadas.

Para esto debemos afirmar que "la educación es un proyecto ético" (Tamayo y Martínez, 1992, p. 31), es decir, pensarnos como sujetos en la sociedad. Entender nuestra realidad y no repetir estructuras que se han petrificado por ciertos intereses de dominación es un objetivo de una educación ética, ya que esta llamada a la constante pregunta y duda.

En este sentido, a partir de la aparición de la llamada historia nueva, como lo indica Betancourt (1995):

comenzaron a ser cuestionadas las viejas corrientes positivistas, en general sostenidas por aficionados refugiados en algunos empleos públicos y en las academias de historia desde donde habían construido una versión "oficial" del pasado colombiano, heroico, elitista y plagado de acontecimientos, y creado una patria. (p. 19)

Iniciativas como estas permiten la posibilidad de cambio de paradigma de la historia, a un cambio de aprendizaje encontrando nuevos discurso que se habían perdido y en este caso del posconflcto colombiano, las posibilidades de una historia narrativa ética permitirá una estabilidad y reconocimiento social de los agentes participantes.

\section{La educación en el posconflicto}

La educación tiene un papel importante en la etapa del posconflicto porque "la educación suministra protección física, psicológica y cognitiva"(Infante, 2013, p. 226), ya que dentro de la educación no tan solo se construye un saber sino una reflexión sobre el entorno.

A partir de lo comentado, la educación en Colombia ha iniciado un proceso de reflexión sobre su papel en la solución de conflictos a partir de programas tales como el Programa Nacional de Competencias Ciudadanas, que busca que estas competencias estén presentes en las áreas del conocimiento en la escuela, para permitir construir procesos de convivencia, pluralidad y participación. 
Se ha querido que estos estándares sean una prioridad en la escuela, ya que buscan formar una ciudadanía con la perspectiva de los derechos humanos. "Las competencias ciudadanas son el conjunto de conocimientos y de habilidades cognitivas, emocionales y comunicativas que, articulados entre sí, hacen posible que el ciudadano actúe de manera constructiva en la sociedad democráticas" $\left(\mathrm{MEN}^{2}, 2003\right.$, p. 8).

También se ha implementado Aulas en Paz, proyecto originario de Canadá, enfocado en el desarrollo de las competencias ciudadanas a partir de tres componentes: "un curriculum, talleres con los padres y talleres con refuerzos con los padres" (Chaux, 2013, p. 500).

Estos proyectos están vinculados con la resolución de problemas; sin embargo, se deben crear espacios de reflexión pedagógica sobre el área de historia del conflicto, ya que esta temática debe vincularse a un proceso de aprendizaje político sobre nuestra sociedad.

Al entender el estudio de la historia y su presencia en la escuela para cumplir un rol de orden social, debemos cambiar paradigmas en nuestra educación y la forma de vernos, puesto que esta posición no dará cuenta de los sucesos de nuestro conflicto a nivel temporal.

Pero para esto debemos definir el concepto de posconflicto, ya que en la actualidad se habla de este como un suceso después de la firma de un proceso de paz. Para Infante (2013, citando a la FRIDE) es el "periodo de tiempo en el cual las hostilidades del pasado se han reducido al nivel necesario para las actividades de reintegración y rehabilitación se pueden iniciar" (p. 228).

Esta definición indica la temporalidad y la disminución de las hostilidades; se pasa de una etapa de baja intensidad militar, donde los contrincantes comiencen un escenario de diálogo, como sucesos posteriores al conflicto, que conduzcan a una legitimidad del estado.

Sin embargo, para Retteberg (2003), esta etapa debe existir con una anticipación de los sucesos ya que "una vasta experiencia internacional enseña que anticipar los temas de los que una sociedad tendrá que ocuparse en el post-conflicto presiona y precipita el fin del conflicto y sienta las bases para una paz duradera" (Rettberg, 2003, p. xix); esto es, es necesario anticiparnos a los acontecimientos y no esperar la etapa posterior.

Esta anticipación de los sucesos parte, por un lado, de una justicia transicional y, por otro lado, de la preparación de la sociedad para la

2 Ministerio de Educación Nacional de Colombia. 
paz. La justicia transicional "se entiende como el esfuerzo por construir paz sostenible tras un período de conflicto, violencia masiva o violación" (Van Zyl, 2011, p. 43), ya que la paz sostenible se basa en un reconocimiento y no en un olvido parcial de la historia para seguir adelante, tampoco se trata de estancarnos sino de reflejarnos como sociedad.

Ahora, la sociedad debe estar dispuesta al proceso de paz, ya que "la construcción de la paz es principalmente un reto y una responsabilidad de carácter nacional, y sólo los actores nacionales pueden abordar de una forma sostenible las necesidades de y las [sic] metas de una sociedad" (Rettberg, 2013, p. xxv).

La paz es una responsabilidad de todas las personas, y la escuela debe construir nuevas formas de pensar y, con estas perspectivas nuevas, podremos construir una cultura del debate (Tamayo y Martínez, 1992).

Es decir, una acción que se puede interpretar como acontecer del presente y del pasado, para indagar estos sucesos, ya que uno de los problemas de un posconflicto es la "verdad" de las partes, cada una de ellas tratará de justificar las acciones cometidas; pero esto no puede llevar a la impunidad.

Esto es importante porque, al finalizar el conflicto, las tareas que se establecen son muchas, pero se vinculan más a nivel económico para reconstruir una base sólida, ya que los conflictos en este sector son por las condiciones económicas y sociales, pero un factor importante es la legitimidad del Estado, por eso se debe evitar la impunidad.

Esto implica la formación permanente de los maestros y maestras en la creación de estrategias para la enseñanza de la historia, que permitan la creación de espacios propicios para que el conflicto pueda ser llevado a una reconciliación, donde el presente y pasado puedan ser vistos de forma crítica.

\section{Conclusión}

Con lo anterior se destaca que el papel de la historia no es neutral y constituye una posibilidad de construir identidad nacional, pero esta está inscrita en interés ideológicos, que puedan causar la construcción de una historia oficial que niega la presencia de otros grupos de la población que no se inscriben en los estamentos de poder.

A partir de esto, el aprendizaje de la historia debe ser una reflexión sobre las múltiples causas que permitieron ciertos sucesos en la 
sociedad, también visibilizar grupos sociales que no han sido nombrados por la historia oficial, permitiendo que el aprendizaje de la historia desarrolle un pensamiento histórico contextualizado y con una lectura global de la realidad.

Con esto se destaca que el posconflicto en Colombia será un proceso arduo de reconciliación, no tan solo se tratará de la firma de un tratado, sino de la posibilidad de entender los sucesos que han generado semejante conflicto. Para esto, la escuela debe tener un discurso pedagógico ético, no que legitime una posición sino que permita diversas posibilidades para entender el posconflicto.

La importancia de la enseñanza de la historia del posconflicto es podernos ver como sociedad y construir democracia entendiendo a la democracia como Zuleta la plantea: "diálogo entre iguales" (Tamayo y Martínez, 1992, p. 35), un diálogo que la escuela debe generar.

Entonces, debemos entender que el posconflicto no se trata de un romanticismo o de un época dorada; es tan solo una posibilidad de transformar nuestra realidad y para esto se necesitan esfuerzos y no simplemente negar los sucedido; para esto la academia debe abrir sus puertas a estos debates, que causen nuevos paradigmas ayudando a legitimar la paz.

Dicha legitimidad debe darse por vías pedagógicas, construidas por los elementos históricos y, para esto, debe entender que la escuela tendrá una tarea ética: construir una propuesta pedagógica posconflicto, la cual será reflexionar sobre qué clase de sociedad quiere y, por tanto, su "verdad" no puede pasar por encima del olvido.

Finalmente, queda claro que es importante pensar en la enseñanza del posconflicto y que esta situación debe ser dada en el presente. Entonces, es un llamado a seguir indagando sobre alternativas y posibilidades para que no se sigan contando historias sin un contexto adecuado.

\section{Referencias}

Adorno, T. (1998). Educación para la emancipación. Madrid: Ediciones Morata.

Álvarez, A. (1995). ... Y la escuela se hizo necesaria: En busca del sentido actual de la escuela. Santafé de Bogotá: Magisterio.

Betancourt, D. (1995). Enseñanza de la historia a tres niveles: Una propuesta alternativa. Bogotá: Magisterio. 
Bloch, M. (1952). Introducción a la historia. México: Fondo de Cultura Económica.

Bordieu, P. (1995). La reproducción: Elementos para una teoría del sistema de enseñanza. Barcelona: Fontamara.

Carretero, M. y Montanero, M. (2008). Enseñanza y aprendizaje de la historia: Aspectos cognitivos y culturales. Revista Cultura y Educación, 20(2), 133-142. Recuperado de http://www.ub.edu/ histodidactica/images/documentos/pdf/ensenanza_aprendizaje_historia.pdf

Carr, E. (1984). ¿Qué es la historia? Barcelona: Planeta Agostini.

Carnoy, M. (2000). La educación como imperialismo cultural. Buenos Aires: Siglo XXI.

Castro, E. (2008). Michel Foucault Perspectiva contemporánea alrededor de su obra. Bogotá: Universidad Libre.

Childe, V. (1985). Qué sucedió en la historia. Bogotá: Planeta Agostini. Durkheim, E. (1976). Educación y sociología. Bogotá. Editores Babel. Infante, A. (2013). El papel de la educación en situaciones de posconflicto: Estrategias y recomendaciones. Revista Hallazgo, 11(21), 223-245.

Lepe, E. y Lima, L. (2014). Estrategias de lectura para comprender relatos históricos en la educación primaria. México: INEE.

Linares, A. (31 de agosto de 2013). Historia, la gran olvidada de las aulas. Periódico El Tiempo.

Ministerio de Educación de Colombia MEN (2003). Estándares básicos de competencia ciudadana. Bogotá: MEN.

Perrone, P. (1999). ¿Por qué necesitamos una pedagogía de la comprensión? En Stone Wiske (Ed.), La enseñanza para la comprensión (pp.35-68). Buenos Aires: Paidós.

Rettberg, A. (compiladora). (2013). Construcción de la paz. Bogotá: Universidad de los Andes.

Rettberg, A. (2003). Diseñar el futuro: Una revisión de los dilemas de la construcción de paz para el post-conflicto. Revista de estudio social, 15.

Touraine, A. (1997). Pourrons-nous vivre ensemble. París: Editorial Fayard. Van Dijk, T.A. (2005). Ideología y análisis del discurso. Utopia y praxis latinoamericana, 10(29), 9-36. Recuperado de http://www.scielo.org.ve/ scielo.php?script=sci_arttext\&pid=S1315-52162005000200002\&1ng=es\&tlng=es. 